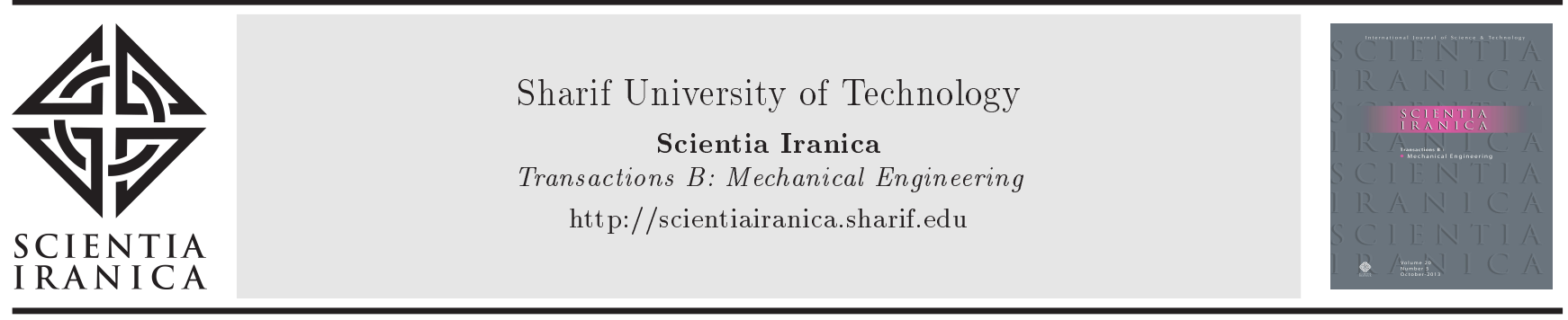

\title{
A novel procedure for micromechanical characterization of white matter constituents at various strain rates
}

\author{
H. Hoursan, F. Farahmand, and M.T. Ahmadian* \\ School of Mechanical Engineering, Sharif University of Technology, Tehran, P.O. Box 145888-9496, Iran. \\ Received 19 May 2018; received in revised form 8 October 2018; accepted 19 November 2018
}

\author{
KEYWORDS \\ Characterization; \\ Hyperelastic \\ coefficients; \\ Representative volume \\ element; \\ Finite element; \\ Periodic boundary \\ conditions; \\ Strain rate.
}

\begin{abstract}
Optimal hyperplastic coefficients of the micromechanical constituents of the human brain stem were investigated. An evolutionary optimization algorithm was combined with a Finite Element (FE) model of a Representative Volume Element (RVE) to find the optimal material properties of axon and Extra Cellular Matrix (ECM). The tension and compression test results of a previously published experiment were used for optimizing the material coefficients, and the shear experiment was used for the validation of the resulting constitutive model. The optimization algorithm was used to search for optimal shear moduli and fiber stiffness of axon and ECM by fitting the average stress in the axonal direction with the results of the experiment. The resulting constitutive model was validated against the shear stress results of the same experiment, showing strong agreement. The instantaneous shear moduli and fiber stiffness of both axon and ECM increased at higher strain rates, while the axon-to-ECM shear modulus ratio decreased from the value of 10 at a strain rate of $0.5 / \mathrm{s}$ to the value of 5 at a strain rate of $30 / \mathrm{s}$. The proposed characterization procedure and the resulting coefficients may be applied to future multi-scale FE studies of the human brain.
\end{abstract}

(C) 2020 Sharif University of Technology. All rights reserved.

\section{Introduction}

Diffuse Axonal Injury (DAI) is a severe type of Traumatic Brain Injury (TBI). This phenomenon is characterized by microscopic damage to brain axons in a vast area of white matter including focal damage to the axons of the corpus callosum and brain stem. The outcome is usually coma, with over $90 \%$ of patients with severe DAI never regaining consciousness [1]. Few survivors most often end up with poor neurological functions as a result of delayed secondary axon dis-

*. Corresponding author. Tel.: +982166165503

E-mail addresses: Hesam.hoursan74@student.sharif.edu (H.

Hoursan); Farahmand@sharif.edu (F. Farahmand);

Ahmadian@sharif.edu (M.T.Ahmadian)

doi: $10.24200 /$ sci.2018.50940.1928 connections, slowly developing over an extended time course [2].

Understanding the mechanical behavior of the brain material is the key to predicting the response of the tissue to external stimuli. The importance of anisotropic behavior of the white matter has been shown in several previous studies [3-6]. The characterization of the anisotropic hyper-viscoelastic material of the white matter can improve the predication and prognosis of TBI. To this end, experimental studies play a central role. Several researchers have performed tests with compressive, tensile, and shear oscillatory loading at various strain rates and specimen geometries to examine the material properties of the brain in vitro. In this respect, among many other valuable publications, the works of Miller and Chinzei (2002) [7], Gefen and Margulies (2004) [8], Hrapko et al. (2006) [9], and Xin et al. (2013) [10] are referenced here. The 
results of in-vitro experimental tests on brain white matter show that the resulting curves depend on several factors including the number of hours post-mortem, the examined region, strain rate, etc. Most of the experiments have been performed at low strain rates corresponding to low-speed phenomena such as tumor growth, surgical procedures, etc., while fewer studies have focused on high strain-rate conditions such as that of TBI. Recently, Budday et al. [6,11] applied a sequence of loading modes to the same human brain specimen and characterized the loading-mode specific regional and directional behaviors. They reported that brain material had a pronounced compression-tension asymmetry.

In the field of FE modeling, a great deal of literature has been dedicated to the macro mechanical and material modeling of the brain tissue (see e.g., [12-16]). However, given the anisotropic nature of white matter discovered as a result of applying the DTI technique and fiber tractography in recent years, multi-scale models have gained considerable importance $[6,17]$. The relation between mechanical loading at a macroscopic head level and cellular damage at a microscopic level is a complex problem. Different length scales involved in the DAI have attracted many researchers to study the multi-scale behavior of white matter $[18,19]$. Since the introduction of the multi-scale models, the micromechanical behavior of the white matter has been under scrutiny due to its anisotropy and heterogeneity, of which the former is represented by axonal bundles extending through white matter in various directions and the latter is the result of regional dependence of axon-to-ECM volume fraction throughout the white matter.

Among multi-scale models of white matter, micromechanical models have gained considerable attention where a Representative Volume Element (RVE) consisting of fiber (axonal bundle) and ECM (ExtraCellular Matrix) is assumed to represent a unit cell within the white matter (e.g., the brain stem). The characterization of such an RVE can serve as a useful tool to shift from a heterogeneous model to a homogeneous model in various sub-regions of the white matter. Several researchers have attempted to characterize the mechanical behavior of an RVE. Ning et al. (2006) [20] developed a composite model with unidirectional fibers to study the behavior of brain stem under finite shear deformation. They used a strain energy function to determine the instantaneous response of the transversely isotropic hyperelastic tissue. Karami and Shankar (2011) [21] proposed a micromechanical composite fibrous RVE to study the homogenized behavior of brain tissue with the nonlinear anisotropic hyperelastic axon and matrix. Abolfathi et al. (2008) [22], Cloots et al. 2013 [23], Javid et al. (2014), and Carlsen and Daphalapurkar (2015) [24,25] are notable among other important works in this area. In some studies, authors have characterized the viscoelastic constants of axon and ECM in the form of Prony series by fitting the constants to a relaxation test $[22,24]$. In some others, the authors have considered hyperelasticity of the tissue and sought to include it in the form of Holzapfel and Fung anisotropic models with constants from the literature [21,23].

Recently, Latorre et al. (2016) [26] studied the effect of considering the compression branch in the characterization of the hyperelastic behavior of soft biological tissues. They concluded that tensile tests were not sufficient to characterize the material behavior of soft biological tissues. By using theoretical formulations for addressing the adequate determination of the terms of stored energies, they showed that, in general, both the extension and compression branches of a uniaxial test were required to completely characterize the material in order to use the obtained model in other loading situations with confidence in the results. In other words, to properly characterize the hyperelastic soft biological tissues, the compression and tension branches of the uniaxial tests (or valid alternative tests) must be considered.

Despite the previous efforts made to characterize the micromechanical behavior of brain tissue, the study of the micromechanical behavior of the white matter constituents (i.e., axon and ECM) has been mostly limited to the small-strain linear viscoelastic response. In addition, to the authors' knowledge, in previous procedures for the hyperelastic characterization of brain tissue, the important effect of the compression branch on the results has been neglected, i.e., the tension test results alone have been used to characterize the material model. The current study seeks to find the optimal hyperelastic constants of the Holzapfel material model [27] for white matter constituents at three characteristic strain rates. This is done by using an evolutionary optimization algorithm to fit the constants of the hyperelastic model with the results of a previous experiment on a specimen at various strain rates. The tension and compression tests are both used to obtain the parameters, and the shear test results are used to validate the resulting model.

\section{Representative Volume Element (RVE)}

RVE is defined as the smallest volume of which a measurement can be made that will yield a value representative of the whole [28]. In case of brain white matter, this volume must contain the extra-cellular matrix and at least one fiber (a bundle of axons), which represents the geometry of the region under study [24]. Here, a cubic unit cell with a size of a conventional T1 voxel from MRI Scanning is considered. The axon-ECM volume ratio is obtained from the previous 
observations of post-mortem samples of porcine brain stem. A single-fiber composite FE model is used with perfect bonding between the constituents.

\subsection{Finite Element (FE) model}

FE model involving the geometry of a fiber and matrix comprising a repetitive unit cell was developed. The fiber was modeled as a partition to represent perfect bonding within the matrix. The axonal volume fraction has been reported to be $53 \%$ in two previous studies [3,29] and $52.7 \%$ [24] from the observations of the porcine brain stem. Here, this fraction is assumed to be $53 \%$. Since the model contains a single fiber rather than a random distribution of fibers, the hypothesized size of the RVE does not affect the results of the study. Here, The RVE edge (characteristic) length is considered $2 \mathrm{~mm}$. On the other hand, the effect of the mesh size on the responses of the RVE is important and must be considered. Therefore, a sensitivity analysis was performed by changing the seed size from 0.01 to $0.2 \mathrm{~mm}$ while observing the average RVE stress at various strain rates. Finally, the seed size of 0.1 $\mathrm{mm}$ was found to be fine enough for stabilizing stress changes at all three strain rates.

The model was meshed with the total number of 1320 linear hexahedral elements of type C3D8R in ABAQUS 6-14. This study used partitioning to obtain fully symmetric mesh on opposite faces.

\subsection{Periodic boundary conditions}

Due to the repetitive nature of the RVE, special boundary conditions known as the Periodic Boundary Conditions (PBC) are required. A number of researchers have shown that the choice of $\mathrm{PBC}$ yields more reasonable results than other boundary conditions such as stress uniform boundary condition or kinematic uniform boundary conditions [30].

The PBC constraints must be applied such that the unit cell deforms in a symmetric and identical manner on the opposite sides. To establish the required symmetry, the number and orientation of nodes on opposite faces of the cell must be equal and similar. The mesh on the opposite surfaces of the RVE must be identical in order for the nodal displacements to have one-to-one correspondence on opposite faces. This will ensure the symmetry of displacements. In order to enforce this condition, the relative displacement of each pair of opposite nodes was formulated in terms of the deformation gradient tensor $(F)$, which is applied to the unit cell in the fiber direction. Since we are dealing with large strains, it is ensured that the displacements conform to the finite strain theory of continuum.

Considering a deformation gradient field acting on the unit cell with the characteristic length $L$, we get:

$$
F_{i j} \quad i, j=1,2,3 \text {. }
$$

For each pair of nodes on two opposite faces (considering the nodes on $S_{+x}$ and $S_{-x}$ as an example), we get:

$$
\begin{aligned}
& u_{S_{+x}}-u_{S_{-x}}-L\left(F_{11}-1\right)=0, \\
& v_{S_{+x}}-x_{S_{-x}}-L F_{12}=0 \\
& w_{S_{+x}}-w_{S_{-x}}-L F_{13}=0
\end{aligned}
$$

where $u, v$, and $w$ are the nodal displacements in $x, y$, and $z$ directions, respectively (Figure 1 ). For each node on an edge, there are two sets of constraint equations to be satisfied since there are two adjacent edges and two corresponding nodes. Taking a node on the edge $E_{S_{-x} S_{+z}}$ as an example, we get:

$$
\begin{aligned}
& u_{E_{S_{+x} S_{+} z}}-u_{E_{S_{-x} S_{+} z}}-L\left(F_{11}-1\right)=0, \\
& v_{E_{S_{+x} S_{+z}}}-v_{E_{S_{-x} S_{+}}}-L F_{12}=0 \\
& w_{E_{S_{+x} S_{+z}}}-w_{E_{S_{-x} S_{+z}}}-L F_{12}=0 .
\end{aligned}
$$

The second set can be obtained by changing the sign of z-plane and rewriting the strains for direction 3 as follows:

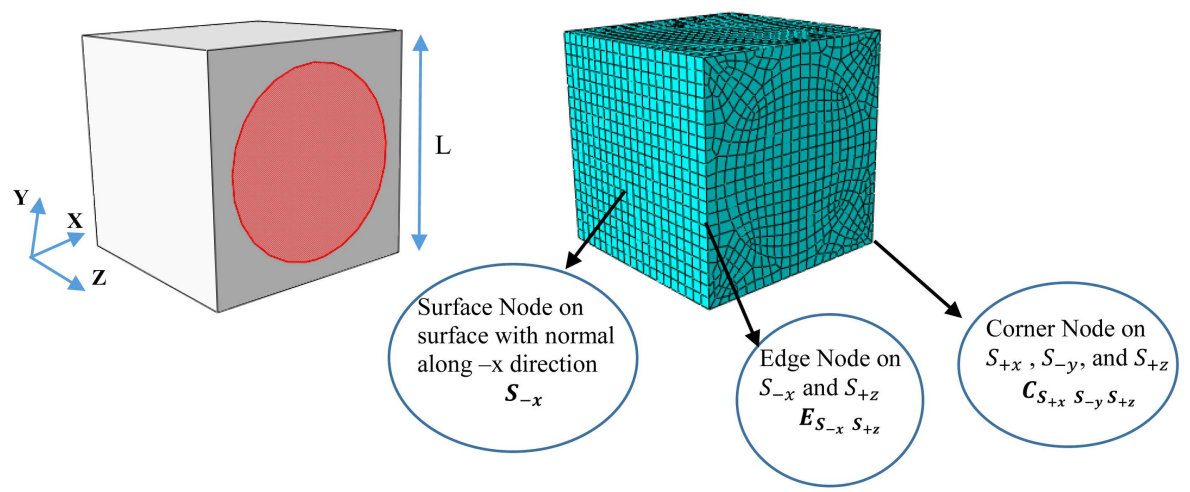

Figure 1. The micromechanical RVE consisting of axon and ECM with a volume ratio of $53 \%$ (left). Meshed FE model of the RVE with an illustration of surface, edge, and corner nodes. The normal vectors of the RVE faces are assumed outward. 


$$
\begin{aligned}
& u_{E_{S_{-x} S_{+} z}}-u_{E_{S_{-x} S_{-} z}}-L F_{13}=0, \\
& v_{E_{S_{-x} S_{+} z}}-v_{E_{S_{-x} S_{-} z}}-L F_{23}=0, \\
& w_{E_{S_{-x} S_{+} z}}-w_{E_{S_{-x} S_{-} z}}-L\left(F_{33}-1\right)=0 .
\end{aligned}
$$

The same approach was applied to the corner nodes. However, since each corner node is opposite to three other corner nods, three sets of equations (9 equations) govern the displacements of a corner node. For brevity, the equations for displacement of the corner node, $u_{C_{S_{+x} S_{+y} S_{+z}}}$, along $x$ axis $(u)$ are written here:

$$
\begin{aligned}
& u_{C_{S_{+x} S_{+y} S_{+z}}}-u_{C_{S_{-x} S_{+y} S_{+z}}}-L\left(F_{11}-1\right)=0,
\end{aligned}
$$

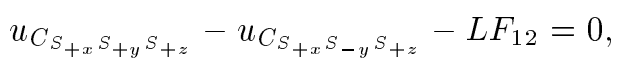

$$
\begin{aligned}
& u_{C_{S_{+x} S_{+y} S_{+z}}}-u_{C_{S_{+x} S_{+y} S_{-z}}}-L F_{13}=0 .
\end{aligned}
$$

Similarly, the remaining equations were derived for displacements of the corner nodes along $y$ and $z$ axes. The above equations were generated via a python script. The redundant constraints were removed at the end.

\subsection{Volume averaging}

Volume averaging was applied to the stress and strain over the RVE to obtain homogenous results [31]. An ABAQUS user subroutine was used to integrate the nodal stress and strains over the volume of the RVE. Eqs. (14) and (15) were used to estimate volumeaveraged values of the outputs:

$$
\begin{aligned}
& \bar{\sigma}_{i j}=\frac{1}{V} \int_{V} \sigma_{i j}(x, y, z) d V, \\
& \bar{\varepsilon}_{i j}=\frac{1}{V} \int_{V} \varepsilon_{i j}(x, y, z) d V,
\end{aligned}
$$

where $\bar{\sigma}_{i j}$ and $\bar{\varepsilon}_{i j}$ represent the volume-averaged values of stress and strain, respectively. $\sigma_{i j}$ and $\varepsilon_{i j}$ are the average nodal stress and strain, respectively, and $V$ is the volume of the RVE.

\section{Material properties}

In order to analyze the large-strain response of the tissue at various strain rates, a hyper-viscoelastic model was used for both axon and ECM. The HolzapfelGasser-Ogden (HGO) strain energy function was used to model the hyperelastic behavior of the constituents. In addition, both axon and ECM were assumed to display the viscoelastic behavior characterized by Prony series constants from a previous experiment.

\subsection{Hyper elastic constitutive model}

The form of the strain energy potential is based on that proposed by Holzapfel et al. (2000) [27]. The HGO model was originally developed for modeling arterial layers with distributed collagen fiber orientations. However, later, it was generalized to account for the fiber dispersion in brain white matter [25]. Wright and Daphalapurkar (2013) [18] and Giordano et al. (2014) [32] demonstrated the application of a simplified version of the HGO model to the brain by reducing the number of material parameters that can be characterized from available experiments. The general form of the function can be written as follows:

$$
\begin{aligned}
W= & \frac{G}{2}\left(\overline{I_{1}}-3\right)+K\left(\frac{J^{2}-1}{4}-\frac{1}{2} \ln J\right) \\
& +\frac{k_{1}}{2 k_{2}} \sum_{i=1}^{n}\left(e^{k_{2}<\bar{E}_{i}>^{2}}-1\right),
\end{aligned}
$$

where:

$$
\bar{E}_{i}=\kappa\left(\overline{I_{1}}-3\right)+(1-3 \kappa)\left(\overline{I_{4 l}}-1\right),
$$

where $W$ denotes the strain energy per unit volume; $G$ and $K$ are the shear and bulk moduli, respectively; $n$ is the number of existing fiber families in the fibermatrix composite model; $\overline{I_{1}}$ is the first invariant of the isochoric Cauchy-Green deformation tensor; $J=$ $\operatorname{det}(F)$ is the volume ratio; and $\overline{I_{41}}=\bar{C}: \vec{n}_{0 i} \vec{n}_{0 i}$, where $\bar{C}=J^{\frac{-2}{3}} C$ is the isochoric part of the right CauchyGreen deformation tensor and $\vec{n}_{0 i}$ stands for the unit vector of fiber direction in the reference coordinate system. The material parameter, $K$, describes the dispersion of the fiber orientations around the preferred fiber direction, $\vec{n}_{0 i}$. $K$ is zero for fully aligned fibers denoting full transverse isotropy and $\frac{1}{3}$ for isotropic fiber orientation (randomly oriented fibers). The Macaulay brackets cause $\bar{E}_{i}$ to become zero in case its value is negative and, thus, the fibers do not contribute to compression.

By assuming a single direction for fibers in the above equation and neglecting nonlinear stiffness effects of the fiber $\left(k_{2} \rightarrow 0\right)$, Eq. (17) is reduced to the following:

$$
W=\frac{G}{2}\left(\overline{I_{1}}-3\right)+K\left(\frac{J^{2}-1}{4}-\frac{1}{2} \ln J\right)+\frac{k_{1}}{2}\left\langle\bar{E}_{1}\right\rangle^{2} .
$$

This study used the HGO constitutive model for both axon (fiber) and ECM, except that the ECM is assumed to have isotopically oriented fibers (i.e., $\kappa=\frac{1}{3}$ ), while fiber is unidirectionally anisotropic (i.e., $\kappa=0$ ). The bulk modulus is assumed constant and equal to $2.5 \mathrm{GPa}$ for both partitions. There is a limited amount of data in the literature on shear moduli of axon and ECM in the brain stem. Under small deformation, Arbogast and Margulies (1999) [3] 
Table 1. Upper and lower bounds of Holzapfel hyperelastic constants for axon and ECM used in the optimization process.

\begin{tabular}{ccccc}
\hline & $\begin{array}{c}\text { Instantaneous shear } \\
\text { modulus }(\mathbf{k P a})\end{array}$ & $\begin{array}{c}\text { Bulk modulus } \\
(\mathbf{G P a})\end{array}$ & $\begin{array}{c}\text { Fiber stiffness, } \boldsymbol{k}_{\mathbf{1}} \\
(\mathbf{k P a})\end{array}$ & $\begin{array}{c}\text { Dispersion parameter } \\
(\boldsymbol{\kappa})\end{array}$ \\
\hline Axon & 0.1 to 10 & 2.5 & 5 to 1000 & 0 \\
ECM & 0.01 to 5 & 2.5 & 0.1 to 500 & $\frac{1}{3}$ \\
\hline
\end{tabular}

reported the instantaneous elastic moduli of axon and ECM as 63.98 and $23.19 \mathrm{kPa}$, respectively. Javid et al. (2014) [24] reported the values of the same constituents as 12.86 and $4.29 \mathrm{kPa}$, respectively. Ning et al. (2006) [20] computed the average initial shear modulus of the brainstem matrix of 4 -week-old pigs to be $12.7 \mathrm{~Pa}$. The fiber stiffness $\left(k_{1}\right)$ of the Hozapfel model is reported as $11.5 \mathrm{kPa}$ for the axonal tissue by Cloots et al. (2013) [23]. The optimization algorithm will search for the instantaneous shear moduli $(G)$ and the fiber stiffness $\left(k_{1}\right)$ of axon and ECM at three different strain rates. First, the optimal shear moduli are obtained by fitting the stress response to the compression test, followed by using tension tests to obtain the optimal fiber stiffness for both constituents. The upper and lower bounds of the parameters are defined based on the available data in the literature (Table 1).

\subsection{Viscoelastic behavior}

Viscoelasticity may be introduced into the hyperelastic model in the form of Prony series by using [23]:

$$
S(t)=\int_{0}^{t}\left[M_{0}-\sum_{i} M_{i} e^{\left(-\frac{t-p}{\tau_{i}}\right)}\right] \frac{d S_{e}}{d p} d p
$$

where $p$ is the time variable that spans from the initial time to the current time. $S$ is the current deviatoric second Piola-Kirchhoff stress tensor, and $S_{e}$ is the deviatoric elastic second Piola-Kirchhoff stress tensor as derived from the non-volumetric part of Eq. (18), and $M_{0}$ is the instantaneous parameter representing
Table 2. The proposed viscoelastic parameters of axon and ECM in brain white matter [24].

\begin{tabular}{ccccc}
\hline & $\boldsymbol{M}_{\mathbf{1}}$ & $\boldsymbol{M}_{\mathbf{2}}$ & $\boldsymbol{\tau}_{\mathbf{1}}(\mathbf{s})$ & $\boldsymbol{\tau}_{\mathbf{2}}(\mathbf{s})$ \\
\hline Axon & 0.6039 & 0.1083 & 0.60097 & 0.49866 \\
ECM & 0.50001 & 0.25986 & 0.00623 & 0.9 \\
\hline
\end{tabular}

the instantaneous role of the material parameters, $G$, and $k_{1} . \quad M_{i}$ and $\tau_{i}$ are time-dependent coefficients obtained from the material response in the relaxation test. Here, the constants are assumed equal to those proposed by Javid et al. (2014) (Table 2) [24].

\section{Methods}

\subsection{Imperialist Competitive Algorithm (ICA)}

The ICA [33] is an evolutionary optimization method. The algorithm can be considered as the social counterpart of Genetic Algorithms (GAs). The algorithm starts with an initial population. Population individuals called country are either colonies or imperialists such that all together form some empires. The algorithm is based on imperialistic competition among these empires. During this competition, weak empires collapse and powerful ones with lower cost take possession of their colonies. Imperialistic competition converges to a state in which there exists only one empire, and its colonies are in the same position and have the same cost as the imperialist (Figure 2).

\subsection{Optimization procedure}

A two-step procedure to achieve optimal curve fitting

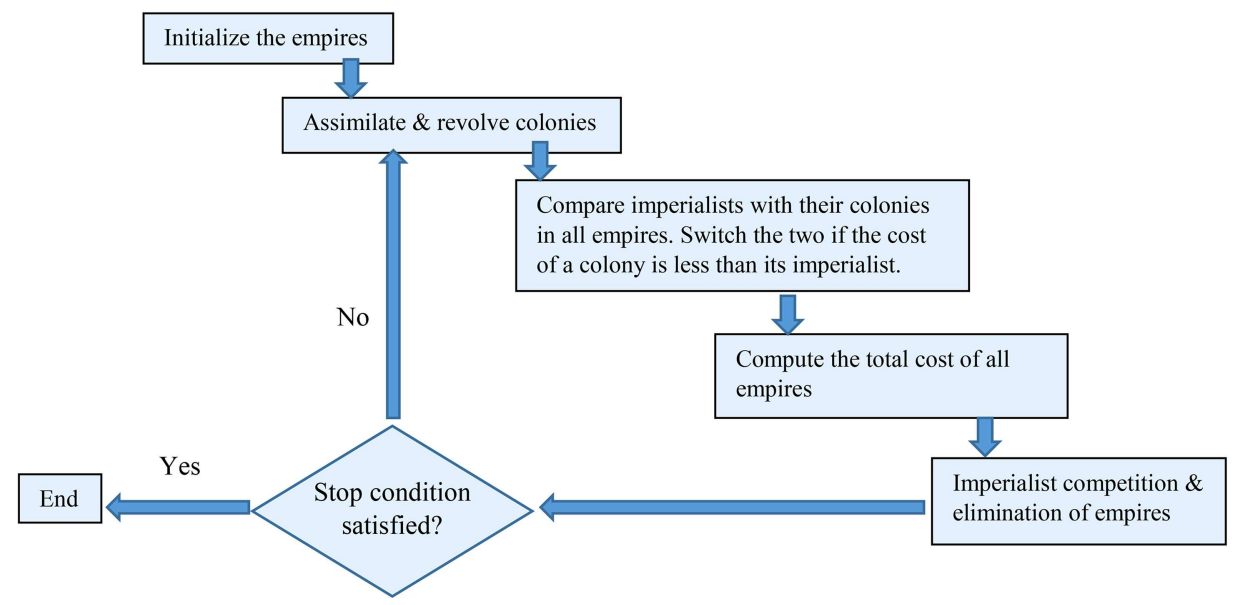

Figure 2. Flowchart of imperialist competitive algorithm [33]. 


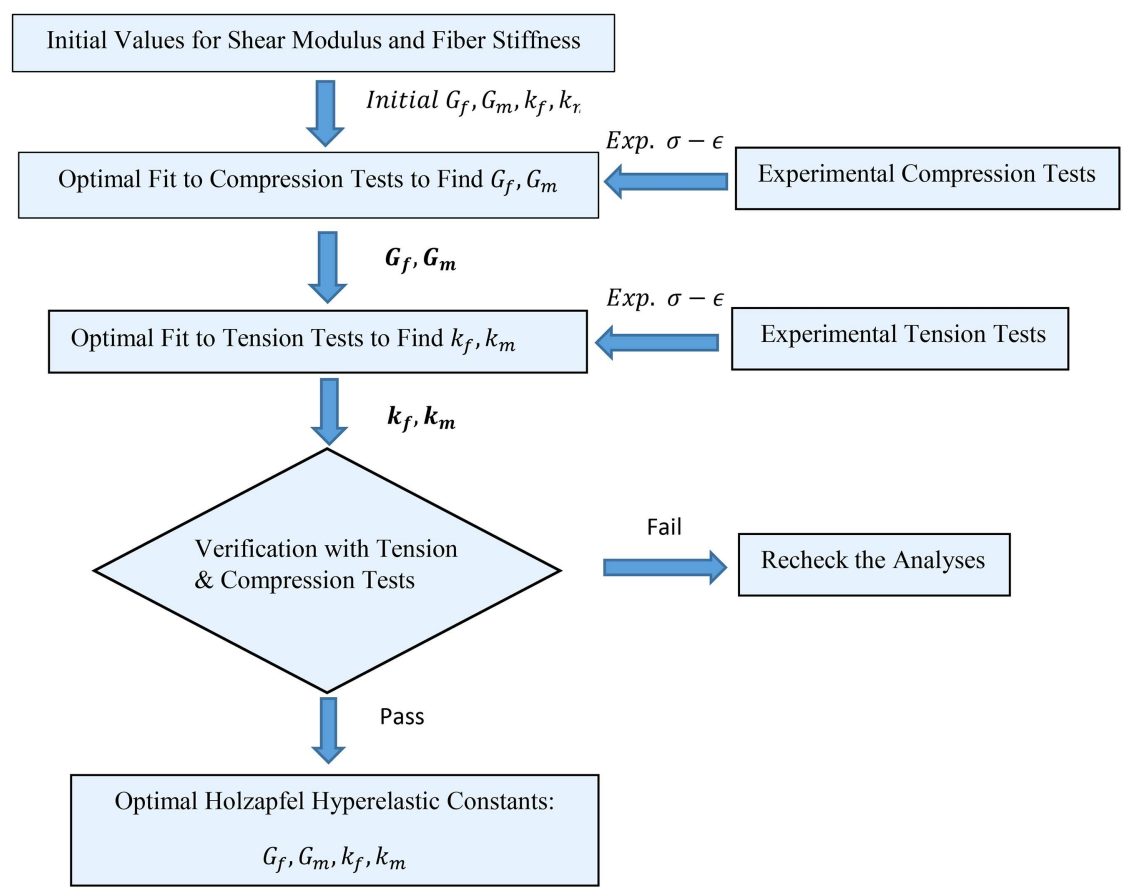

Figure 3. Flowchart of the procedure for finding Holzapfel constants using optimal curve fitting with experimental tension and compression tests.

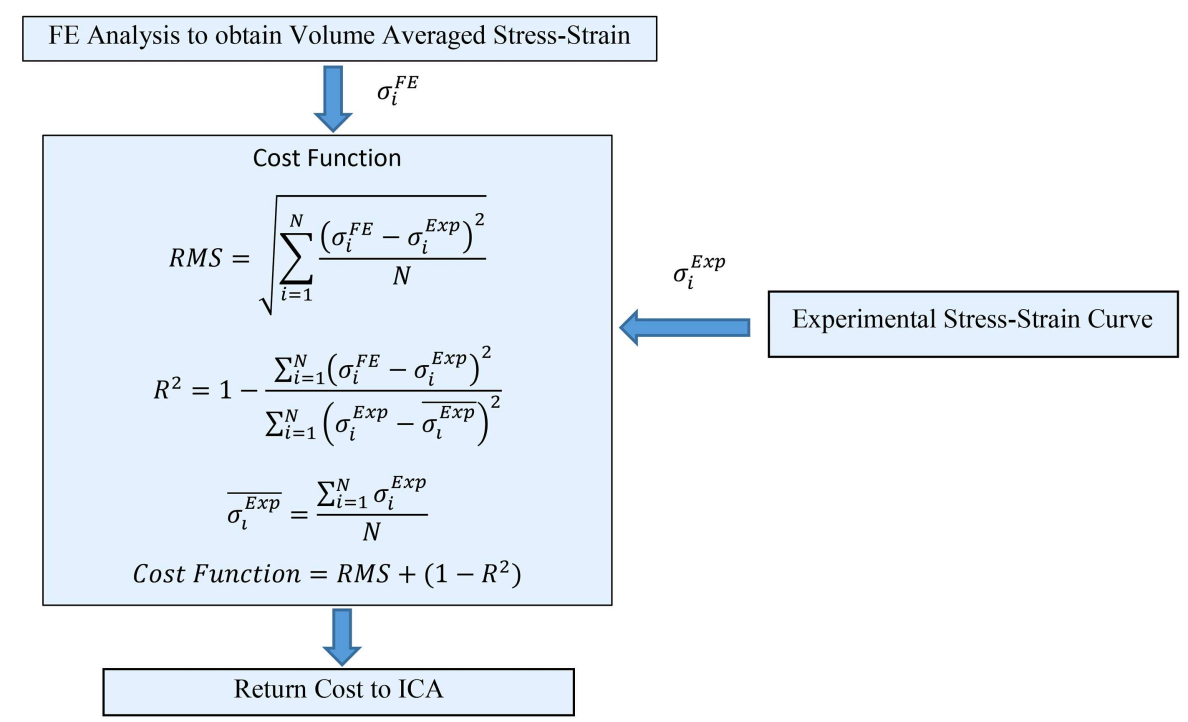

Figure 4. Flowchart of the calculation of costs for each decade of ICA optimization with a Matlab script. $\sigma_{i}^{F E}$ is the volume-averaged stress calculated from the FE RVE model at each increment. $\sigma_{i}^{E x p}$ is the experimental stress from Xin et al. (2013) discretized over the same number of increments $(N)$ as the calculated stress.

was implemented. In the first step, the shear moduli were obtained by fitting the model results with compression test results. In the second step, the fiber stiffness values were found by implementing the previous step (shear moduli) and fitting the model results with tension test results. Finally, the full model was compared by both tests (i.e., tension and compression) to verify the coefficients (Figure 3 ). The cost function was defined as the sum of R2 (coefficient of determination) and RSME (Root Mean Square
Error) of stress values to account for qualitative and quantitative errors of the obtained curves, respectively (Figure 4). The stop condition is defined as a point where only one empire is left as a result of imperialist competition. This resulted in a different number of decades (iterations) before achieving convergence at each run of the algorithm. Separate MATLAB scripts were used to edit the material data of the python script, run the python script in ABAQUS, and get the results from ABAQUS ASCII output file at each run. 
Table 3. Optimal Holzapfel hyperelastic material constants of axon and ECM of brain stem for three strain rates. Shear moduli and fiber stiffness are instantaneous values.

\begin{tabular}{|c|c|c|c|c|c|c|}
\hline \multirow[b]{2}{*}{ Strain rate } & \multirow[b]{2}{*}{$\begin{array}{c}\text { Axon shear } \\
\text { modulus } \\
(\mathbf{k P a})\end{array}$} & \multirow[b]{2}{*}{$\begin{array}{c}\text { ECM shear } \\
\text { modulus } \\
(\mathrm{kPa})\end{array}$} & \multirow[b]{2}{*}{$\begin{array}{c}\text { Axon fiber } \\
\text { stiffness } \\
(\mathbf{k P a})\end{array}$} & \multirow[b]{2}{*}{$\begin{array}{c}\text { ECM fiber } \\
\text { stiffness } \\
(\mathbf{k P a})\end{array}$} & \multicolumn{2}{|c|}{ Minimum cost $\left[R^{2} R S M E\right]$} \\
\hline & & & & & Tension & Compression \\
\hline Low $(0.5 / \mathrm{s})$ & 1.001301 & 0.1011852 & 293.063 & 4.892 & $\begin{array}{c}0.1174 \\
{[0.990 .1074]}\end{array}$ & $\begin{array}{c}0.3242 \\
{[0.96310 .2873]}\end{array}$ \\
\hline Average $(5 / \mathrm{s})$ & 1.823 & 0.241 & 350.43 & 7.86 & $\begin{array}{c}0.0456 \\
{[0.99870 .0443]}\end{array}$ & $\begin{array}{c}0.1881 \\
{[0.97310 .1612]}\end{array}$ \\
\hline High $(30 / s)$ & 4.2162 & 0.80099 & 410.955 & 8.21200 & $\begin{array}{c}0.1983 \\
{[0.98360 .1818]}\end{array}$ & $\begin{array}{c}0.1023 \\
{[0.98890 .0912]}\end{array}$ \\
\hline
\end{tabular}

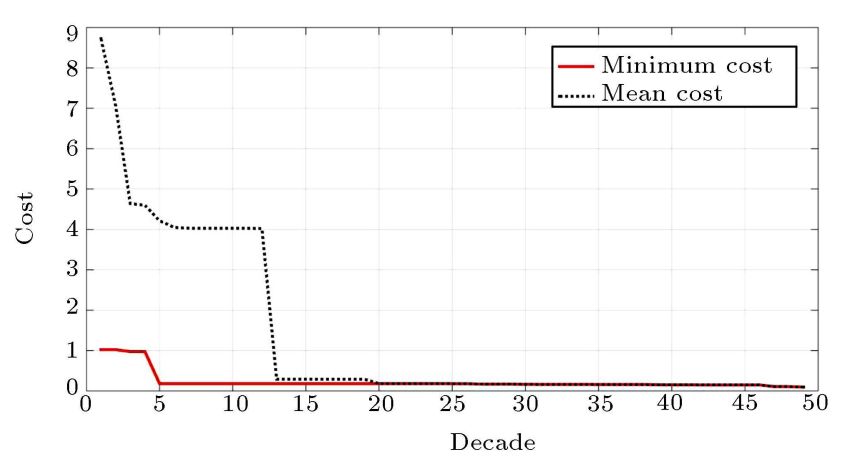

Figure 5. Plot of mean and minimum cost of all countries with decades of ICA algorithm for average strain rate analysis in tension. The algorithm converged after 48 decades with a minimum cost of 0.04 .

For tension test at the average strain rate $(5 / \mathrm{s})$, the algorithm was processed in ABAQUS 320 times and converged after 11 hours and 48 decades (Figure 5 ).

\section{Results}

The optimal hyperelastic parameters in brain stem were found by using the ICA optimization algorithm together with several scripts for calculating costs at each decade (iteration). Ten initial countries and four initial imperialists were selected for the generation of the first set of parameters. The algorithm was repeated for three strain rates, namely low $(0.5 / \mathrm{s})$, medium $(5 / \mathrm{s})$, and high $(30 / \mathrm{s})$ strain rates. The total strain of 0.5 was applied in the fiber direction within the step times of $1,0.1$, and 0.0167 seconds, respectively.

For the three cases, at each run, the volume averaged stress values were requested at 30 increments via a python script and written into an ABAQUS Inp file. The file was then run by a MATLAB script, and the results associated with the cost calculation at every decade were obtained. The iteration was repeated until a single empire was left, and the minimum cost was found as a result. The results of the four Holzapfel hyperelastic parameters for three strain rates are shown in Table 3. Figure 5 shows the convergence of mean

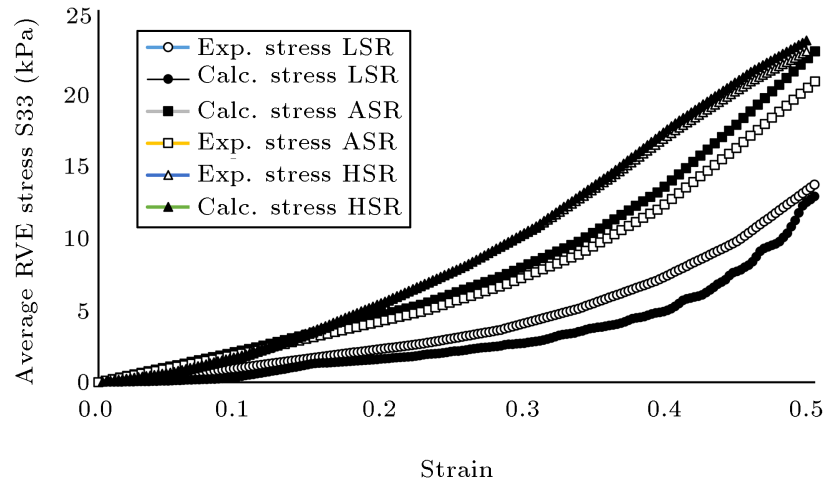

Figure 6. Optimized calculated average stress in fiber direction versus strain in comparison with the experimental data for compression. Compressive strain of 0.5 is applied at three strain rates: LSR: Low Strain Rate $(0.5 / \mathrm{s})$, ASR: Average Strain Rate $(5 / \mathrm{s})$, and HSR: High Strain Rate: $30 / \mathrm{s}$. Experimental curves of white matter samples from Xin et al. (2013) [10].

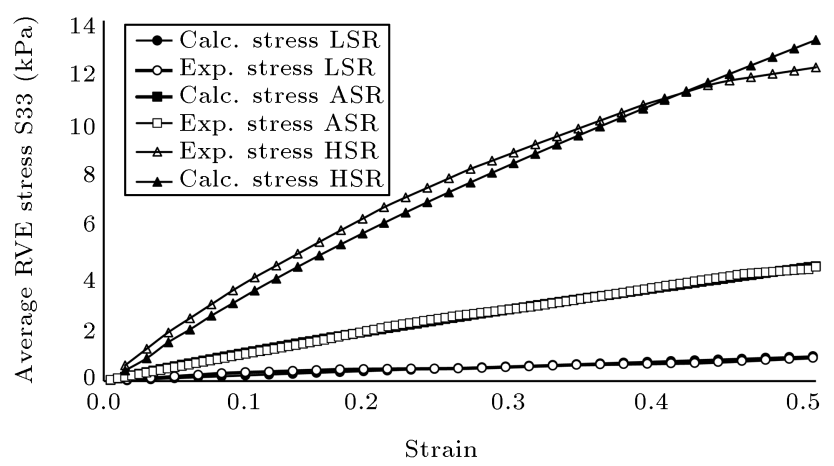

Figure 7. Optimized calculated average stress in fiber direction versus strain in comparison to the experimental data for tension at three strain rates: LSR: Low Strain Rate $(0.5 / \mathrm{s})$, ASR: Average Strain Rate $(5 / \mathrm{s})$, and HSR: High Strain Rate: 30/s. Experimental curves of white matter samples from Xin et al. (2013) [10].

cost of all empires and the minimum cost at 48 decades during a run from the average strain rate scenario. The obtained curves with minimum cost are plotted against experimental data in Figures 6 and 7. 


\subsection{Validation with shear experiment}

The optimal hyperelastic constants of Table 3 may now be validated according to the shear experiments of the referenced experimental study. For this purpose, after applying the obtained optimal constants of the HGO model and using the Prony series constants for viscoelasticity, the shear stress of 0.5 was applied to the RVE in the XZ plane (Figure 1) and the results were obtained (Figure 8). The results show acceptable quantitative agreement (RSME values in figure) and excellent qualitative similarity of the results ( $R 2$ values in figure).

\section{Discussion}

The main goal of this study is to find the optimal hyperelastic constants of the HGO composite model

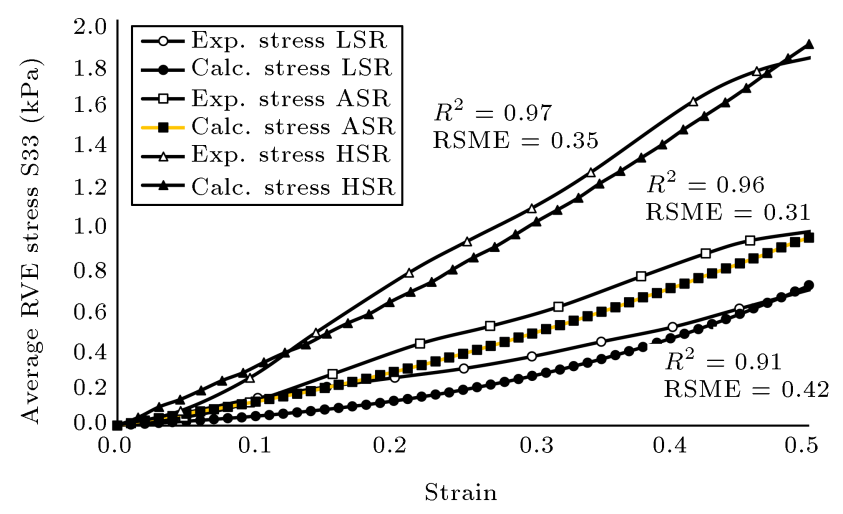

Figure 8. Optimized calculated stress in fiber direction versus strain in comparison to the experimental data for shear. Shear strain of 0.5 is applied at three strain rates: LSR: Low Strain Rate (0.5/s), ASR: Average Strain Rate (5/s), and HSR: High Strain Rate: 30/s. Experimental curves of white matter samples from Xin et al. (2013) [10]. for the brain stem. To this end, an evolutionary global optimization algorithm was used. The timedependent viscoelastic constants were obtained from a previous relaxation test and included in the model in the form of Prony series. The optimization algorithm searched for HGO hyperelastic constants that could best fit the existing experimental curves of tension and compression. This is based on an important notion that one uniaxial test is not enough to characterize the hyperelastic behavior of soft biological tissues [26]. The shear moduli of axon and matrix were obtained from fitting the compression test data and, then, the Holzapfel fiber stiffness values were found by fitting the tension data. The results were obtained for three strain rates, namely the low $(0.5 / \mathrm{s})$, average $(5 / \mathrm{s})$, and high $(30 / \mathrm{s})$ strain rates. The optimal curve fitting algorithm converged in various iterations (decades) in the range of 30 to 100 with the initial population (number of countries) of 10 in all simulations. Finally, the obtained models were validated against the shear experimental data of the same referenced article. The results showed strong agreement both in trend and quantity of the values, showing the reliability of the HGO hyperelastic model for the simulation of the tissue behavior (Figures 6-8). The fiber-direction stress contours of the deformed RVE in three load cases (i.e., tension, compression, and shear) are shown in Figure 9. As can be seen, the stress values are several times higher in axon than the ECM in tension and compression. The symmetry of the stress distribution throughout the RVE can be observed in the shear stress contour, which occurs as a result of the applied periodic boundary conditions.

Ning et al. (2006) [20] reported that the initial shear modulus of axons was 10 times larger than that of ECM under large deformation. This is in

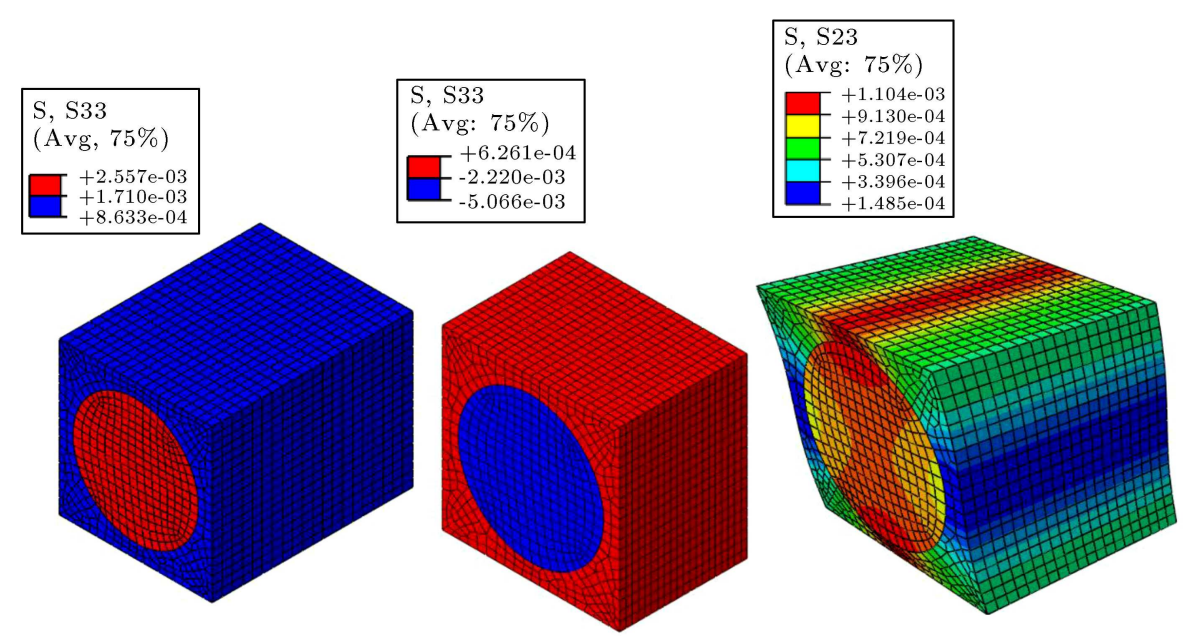

Figure 9. Deformed contour of stress (MPa) for the low-strain-rate scenario at three modes of tension (left), compression (center), and shear (right). The total strain of 0.5 ramped to $1 \mathrm{~s}$. In case of tension and compression tests, normal stress is shown, while the shear stress in the same plane of the applied strain (S23) is shown in the shear test. 
agreement with the results of our study at low strain rates. However, this ratio decreases to 5 at higher strain rates. Rashid et al. (2012) [34] used a high rater tension device to characterize the overall white matter hyperelastic behavior of the porcine brain using various hyperelastic models. They reported the tensile engineering stress values at $30 \%$ strain as $3.1,4.3$, and $6.5 \mathrm{kPa}$ at strain rates of 30,60 , and $90 / \mathrm{s}$, respectively. In the current study, the average RVE stress at $50 \%$ strain and a strain rate of $30 / \mathrm{s}$ was $4.5 \mathrm{kPa}$, which is in agreement in the order of magnitude. However, it should be noted that the tension and compression tests have both been used in the current study, while only the extension of the tissue was considered in the study of Rashid and Ning. In addition, shear moduli of porcine white matter were evaluated by Rashid et al. (2012) [34] in Fung, Gent, and Ogden hyperelastic models at a strain rate of $30 / \mathrm{s}$ to be approximately 3 $\mathrm{kPa}$. The proposed algorithm found the shear moduli of axon and ECM at the same strain rate to be 4.2 and $0.8 \mathrm{kPa}$, respectively. Assuming that the tissue shear modulus is the average of those of axon and ECM, the obtained values of our findings are in good agreement with those of the referenced studies.

Miller and Chinzei (2002) [7] concluded that brain's stiffness in compression was $20 \%$ higher than that in tension. This result, which our model verifies, may imply that axons contribute to the tissue's behavior in compression through an unknown mechanism. This is important given the fact that fibers represent a large portion of brain stem (e.g., $90 \%$ optic fiber volume fraction in the composites of optic fiber-matrix of the adult porcine brainstem [3].

\section{Conclusions}

The focus of this study was mainly to introduce a set of (HGO) hyperelastic constants for the constituents of the brain stem. The constants were obtained by using an evolutionary optimization algorithm for three strain rates. The algorithm searched for constants that could best fit the resulting average stress curve with a previous experiment. Based on the nature of bio-tissues that required more than one test to be characterized, both compression and tension test results were used to reach the constants. The resulting hyperelastic model was validated against the shear test data of the same experiment. It was observed that the results of applying shear strain to the model were in acceptable agreement with those of the experiment. On the other hand, the obtained shear moduli of axon and ECM obtained from the optimization algorithm were in the range of the previously reported values for the anisotropic white matter tissue. The fiber stiffness values of axon were higher than those of the ECM, as expected.
The effect of the compression branch of uniaxial tests in the characterization of hyperelastic models of brain is an important notion. Although it is assumed in the hyperelastic constitutive models that fibers do not contribute to compression, the results of this study and some previous studies such as those of Miller and Chinzei (2002) [7] and Latorre et al. (2016) [26] may challenge this idea.

The current study used a single-directional composite model to simulate the transversely isotropic behavior of the brain stem. This is a basic simplification of the reality of the white matter anisotropy. In addition, in the application of Periodic Boundary Conditions, the large strain assumption was used and the equations were derived based on the finite strain theory. This will result in stresses in the form of the second Piola-Kirchhoff stress tensor. Finally, in the case of (TBI), strain rates of up to $90 / \mathrm{s}$ have been reported in the literature. Future studies may expand the current method to investigate the hyperelastic behavior of the tissue at higher strain rates.

\section{Acknowledgement}

This paper is supported by INSF.

\section{References}

1. Wasserman, L., Shaw, T., Vu, M., Ko, C., Bollegala, D., and Bhalerao, S. "An overview of traumatic brain injury and suicide", Brain Injury, 22(11), pp. 811-819 (2008). DOI: 10.1080/02699050802372166

2. Johnson, V.E., Stewart, W., Smith, D.H. "Axonal pathology in traumatic brain injury", Experimental Neurology, Special Issue: Axonal Degeneration, 246, pp. 35-43 (2013). DOI:10.1016/j.expneurol. 2012.01 .013

3. Arbogast, K. and Margulies, S. "A fiber-reinforced composite model of the viscoelastic behavior of the brainstem in shear", J. Biomech., 32, pp. 865-870 (1999)

4. Arbogast, K.B. and Margulies, S.S. "Material characterization of the brainstem from oscillatory shear tests", J Biomech, 31, pp. 801-807 (1998).

5. Prange, M.T. and Margulies, S.S. "Regional, directional, and age-dependent properties of the brain undergoing large deformation", J Biomech Eng, 124, pp. 244-252 (2002).

6. Budday, S., Sommer, G., Holzapfel, G.A., Steinmann, P., and Kuhl, E. "Viscoelastic parameter identification of human brain tissue", Journal of the Mechanical Behavior of Biomedical Materials, 74, pp. 463-476, ISSN 1751-6161 (2017).

7. Miller, K. and Chinzei, K. "Mechanical properties of brain tissue in tension", J. Biomech., 35, pp. 483-490 (2002). 
8. Gefen, A. and Margulies, S. "Are in vivo and in situ brain tissues mechanically similar?", Journal of Biomechanics, 37, pp. 1339-52 (2004). 10.1016/j.jbiomech.2003.12.032

9. Hrapko, M., Dommelen, J.A.W., Peters, G.W.M., and Wismans, J.S.H. "The mechanical behavior of brain tissue: large strain response and constitutive modeling", Biorheology, 43, pp. 623-636 (2006).

10. Xin, J., Feng, Z., Haojie, M., Ming, S., and King, H. "A comprehensive experimental study on material properties of human brain tissue", Journal of Biomechanics, 46(16), pp. 2795-2801, ISSN 0021-9290 (2013).

11. Budday, S., Sommer, G., Birkl, C., Langkammer, C., Haybaeck, J., Kohnert, J., Bauer, M., Paulsen, F., Steinmann, P., Kuhl, E., and Holzapfel, G.A. "Mechanical characterization of human brain tissue", Acta Biomater., 48, pp. 319-340 (2016). https://doi.org/10.1016/j.actbio.2016.10.036

12. Eslaminejad, A., Hosseini Farid, M., Ziejewski, M., Karami, G. Brain "Tissue constitutive material models and the finite element analysis of blast-induced traumatic brain injury", Scientia Iranica, 25(6), pp. 31413150 (2018). DOI: $10.24200 /$ sci.2018.20888

13. Saboori, P. and Sadegh, A. "Material modeling of the head's subarachnoid space", Scientia Iranica, 18(6), pp. 1492-1499, ISSN 1026-3098 (2011).

14. Hoursan, H., Ahmadian, M., Barari, A., and Naghibi, $H$. "Modelling and analysis of the effect of angular velocity and acceleration on brain strain field in traumatic brain injury", ASME International Mechanical Engineering Congress and Exposition, Proceedings (IMECE), 3 (2013). 10.1115/IMECE2013-63053

15. Shafiee, A., Ahmadian, M., Hoursan, H., and Hoviattalab, M. "Effects of linear and rotational acceleration on human brain", Journal of Mechanical Engineering Modares, 15, pp. 248-260 (2015).

16. Hoursan, H., Ahmadian, M., Kazemiasfeh, R., and Barari, A., On the validity extent of linear viscoelastic models of human brain (2018). 10.25071/10315/35365

17. MacManus, D.B., Pierrat, B., Murphy, J.G., and Gilchrist, M.D. "Region and species dependent mechanical properties of adolescent and young adult brain tissue", Scientific Reports, pp. 2045-2322 (2013). https://doi.org/10.1038/s41598-017-13727-z

18. Wright, R.M., Post, A., Hoshizaki, B., and Ramesh, K.T. "A multiscale computational approach to estimating axonal damage under inertial loading of the head", J Neurotrauma, 30(2), pp. 102-18 (2013). DOI: 10.1089/neu.2012.2418. PubMed PMID: 22992118

19. Goriely, A., Geers, M.G.D., and Holzapfel, G.A. "Mechanics of the brain: perspectives, challenges, and opportunities", Biomechanics and Modeling in Mechanobiology, 14, pp. 931-965 (2015). DOI: $10.1007 / \mathrm{s} 10237-015-0662-4$
20. Ning, X., Zhu, Q., Lanir, Y., and Margulies, S.S. "A transversely isotropic viscoelastic constitutive equation for brainstem undergoing finite deformation", ASME J Biomech Eng, 128(6), pp. 925-933 (2006). DOI:10.1115/1.2354208

21. Karami, G. and Shankar, S. "A multiscale analysis of the white brain material with axons as bidirectional oriented fibers", SIMULIA Customer Conference, pp. 1-14 (2011).

22. Abolfathi, N., Naik, A., Sotudeh, M., Karami, G., and Ziejewski, M. "A micromechanical procedure for characterization of the mechanical properties of brain white matter", Computer Methods in Biomechanics and Biomedical Engineering (In Press). DOI: 10.1080/10255840802430587) (2008).

23. Cloots, R.J.H., Dommelen van, J.A.W., Kleiven, S., and Geers, M.G.D. "Multi-scale mechanics of traumatic brain injury: predicting axonal strains from head loads", Biomechanics and Modeling in Mechanobiology, 12(1), pp. 137-150 (2013). DOI: 10.1007/s10237012-0387-6

24. Javid, S., Rezaei, A., and Karami, G. "A micromechanical procedure for viscoelastic characterization of the axons and ECM of the brainstem", Journal of the Mechanical Behavior of Biomedical Materials, 30, pp. 290-299, ISSN 1751-6161 (2014).

25. Carlsen, R.W. and Daphalapurkar, N.P. "The importance of structural anisotropy in computational models of traumatic brain injury", Front Neurol, 6(28) (2015). DOI: 10.3389/fneur.2015.00028. E-Collection 2015. Review. PubMed PMID: 25745414; PubMed Central PMCID: PMC4333795

26. Latorre, M., De Rosa, E., and Montáns, F. "Understanding the need of the compression branch to characterize hyperelastic materials", International Journal of Non-Linear Mechanics, 89 (2016). 10.1016/j.ijnonlinmec.2016.11.005

27. Holzapfel, G.A., Gasser, T.C., and Ogden, R.W. "A new constitutive framework for arterial wall mechanics and a comparative study of material models", Journal of Elasticity, 61(1), pp. 1-48 (2000). https://doi.org/10.1023/A:1010835316564

28. Hill, R. "Elastic properties of reinforced solids: some theoretical principles", Journal of the Mechanics and Physics of Solids, 11(5), pp. 357-372 (1963). DOI: 10.1016/0022-5096(63)90036-x

29. Meaney, D.F. "Relationship between structural modeling and hyperelastic material behavior: application to CNS white matter", Biomech. Model. Mechanobiol., 1, pp. 279-293 (2003)

30. Stéphane, L. and Stéphane, B. "An Abaqus toolbox for calculation of effective properties of heterogeneous materials", 10th National Symposium in Calculation of Structures, CSMA 2011, 9-13 May, Giens (Var) (2011). 
31. Hollister, S.J. and Kikuchi, N. "A comparison of homogenization and standard mechanics analyses for periodic porous composites", Computational Mechanics, 10(2), pp. 73-95 (1992).

32. Giordano, C., Cloots, R., Dommelen, J., Kleiven, S., and Geers, M. "The influence of anisotropy on brain injury prediction", J Biomech, 47, pp. 1052-9 (2014). Doi: $10.1016 /$ j. j biomech. 2013.12.036

33. Atashpaz-Gargari, E. and Lucas, C. "Imperialist competitive algorithm: An algorithm for optimization inspired by imperialistic competition", IEEE Congress on Evolutionary Computation, 7, pp. 46614666 (2007).

34. Rashid, B., Destrade, M., and Gilchrist, M.D. "Mechanical characterization of brain tissue in tension at dynamic strain rates", Journal of the Mechanical Behavior of Biomedical Materials, 33, pp. 43-54 (2012). 10.1016/j.jmbbm.2012.07.015

\section{Biographies}

Hesam Hoursan is a $\mathrm{PhD}$ graduate at Sharif University of Technology working in the field of traumatic brain injury and multi-scale modeling of soft tissue. He has worked on the same field during his MSc studies as well and has published several articles in the field. His current research focus is multi-scale modeling including macro-micro scale integration in TBI.

Farzam Farahmand is a Professor of Biomechanics and the Deputy of Technology, Sharif University of Technology, Tehran, Iran. He has been the Director of Dr. Javad Mowafaghian Research Center of NeuroRehabilitation Technologies, Tehran, Iran and the Director of a Surgical Robotics Lab, Research Center of Biomedical Technologies Robotics (RCBTR), Tehran University of Medical Sciences, Tehran, Iran. Orthopedic biomechanics, rehabilitation, and soft tissues are the main focus of his studies.

Mohammad Taghi Ahmadian received his BS and MS degree in Physics in 1972 from Shiraz University, Shiraz, Iran and completed the requirements for BS and MS degree in Mechanical Engineering in 1980 from University of Kansas in Lawrence. At the same time, while getting his master's degree, he completed his $\mathrm{PhD}$ in Physics and PhD in Mechanical Engineering in 1981 and 1986, respectively, from University of Kansas. His research interests are micro and nano mechanics and bioengineering. He has recently focused on brain and TBI phenomena as hyper-visco elastic soft tissues. 